\title{
Aging, Hyaluronidase Removal of the Cumulus, and Microinjection Do Not Affect the Sperm Binding Potential of Human Oocytes
}

\author{
ALBERTO BARROS, ${ }^{1}$ MÁRIO SOUSA, $, 2,5$ JOAQUINA SILVA, ${ }^{1}$ VASCO ALMEIDA, ${ }^{3}$ and \\ EDUARDO ROCHA ${ }^{4}$
}

Submitted: August 5, 1996

Accepted: October 10, 1996

Purpose: We studied the influence of aging, hyaluronidase removal of the cumulus, and microinjection on the sperm binding potential of human oocytes under intact zona assay conditions to determine the safe use of unstored aged unfertilized ICSI oocytes on zona binding tests. Results were also compared with those for aged IVF oocytes under the same conditions.

Methods: To avoid the large variation in sperm binding scores, we compared the ratios of the number of sperm bound to nontreated versus to treated oocytes. Treated oocytes were those whose zonae were exposed to hyaluronidase, an agent that decreases sperm binding. Experimental groups were fresh oocytes, experimentally aged fresh oocytes, fresh oocytes whose cumulus was removed either mechanically or with hyaluronidase, aged ICSI oocytes, and aged IVF oocytes.

Results: Statistical analysis within and between groups showed that aging, previous insemination, cumulus removal by hyaluronidase, and microinjection did not affect the sperm binding potential of human oocytes.

Conclusions: As the zona binding ability of fresh and unfertilized aged ICSI oocytes is similar, they can be used safely in zona assays.

KEY WORDS: human; intracytoplasmic sperm injection; zona binding assay.

\footnotetext{
'Laboratory of Medical Genetics, Faculty of Medicine, University of Porto, Porto, Portugal.

${ }^{2}$ Laboratory of Cell Biology, Institute of Biomedical Sciences, University of Porto, Porto, Portugal.

${ }^{3}$ Institute of Anthropology, Faculty of Sciences, University of Porto, Porto, Portugal.

${ }^{4}$ Laboratory of Histology and Embryology, Institute of Biomedical Sciences, University of Porto, Porto, Portugal.

${ }^{5}$ To whom correspondence should be addressed at Laboratory of Cell Biology, Institute of Biomedical Sciences, University of Porto, Lg. Prof. Abel Salazar 2, 4050 Porto, Portugal.
}

\section{INTRODUCTION}

The human sperm-zona pellucida binding test (ZBT) has been developed using a hemizona (1-3) as well as an intact zona (4-6) model. This test has been used either to investigate the molecular mechanisms of human gamete recognition (7-14) or as a clinical assay to determine the potential use of a patient sperm in in vitro fertilization (IVF) attempts (15-20). In these studies, ZBTs have been performed either with fresh immature or mature oocytes or with aged unfertilized IVF oocytes, which were pooled until use in a concentrated salt solution or cryopreserved. In these cases, immaturity, pooling, storage, aging, or previous exposure of the zonae to sperm seemed not to affect the results of the ZBTs adversely (1-20).

Due to the widespread availability of infertility treatments and the use of intracytoplasmic sperm injection (ICSI) (2l-24), some assisted reproduction laboratories now have a large number of unfertilized aged oocytes that come from fertilization failures after ICSI cycles. However, it has not been investigated if hyaluronidase removal of the cumulus and microinjection affect sperm binding.

We usually perform ZBTs using the intact zona model without egg storage by pooling our aged unfertilized oocytes coming from IVF and ICSI cycles. The purpose of the present study was to investigate and compare, under intact zona assay conditions with unstored eggs, the influence of aging, hyaluronidase digestion of the cumulus and corona layers, and microinjection on the subsequent sperm-zona binding ability of human oocytes.

\section{MATERIALS AND METHODS}

Oocytes were recovered from large ovarian follicles by ultrasonic-guided follicular aspiration in cycles 
stimulated with hMG and hCG after pituitary desensitization with a $\mathrm{GnRH}$ agonist and used in a therapeutical IVF or ICSI attempt. The details of these techniques and criteria for patient inclusion were described earlier (21-24).

Mature fresh human oocytes were donated by patients for research purposes when a high number of eggs were recovered during therapeutic IVF/ICSI cycles. Mature unfertilized aged oocytes were those that, $44 \mathrm{hr}$ after IVF/ICSI, showed no signs of fertilization $(23,24)$. Spermatozoa were obtained from samples showing normal values of sperm concentration, motility, and morphology (25). Oocytes and sperm used for experimental purposes were taken after patient consent.

After liquefaction, spermatozoa were washed from seminal plasma with SPM medium (Medicult) by two sequential centrifugation steps of $5 \mathrm{~min}$ at $1000 \mathrm{rpm}$. The final pellet was overlaid with $0.5 \mathrm{ml}$ of IVF medium (Medicult) and incubated for $1 \mathrm{hr}$ at $37^{\circ} \mathrm{C}$ with $5 \% \mathrm{CO}_{2}$ in air to achieve a swim-up separation of vigorously motile spermatozoa.

Fresh oocytes were freed from their cumulus and corona layers either by mechanical means (dissection with fine needles followed by pipetting with fine glass pipettes) or by a 30 -sec period of hyaluronidase (Medicult) treatment. Unfertilized aged ICSI oocytes were already cumulus-free because, before microinjection, the cumulus was removed with hyaluronidase as above. Unfertilized aged IVF oocytes were also already cumulus-free, due in part to having been in culture for $44 \mathrm{hr}$ and also because at $16 \mathrm{hr}$ after insemination the cumulus was partially mechanically removed to search for the presence of pronuclei.

To overcome the problem of the large variation in sperm binding scores, we compared not the number of sperm bound per oocyte but the changes observed in the sperm binding ability of each set of oocytes after priming the zonae with an agent (hyaluronidase) that decreases sperm binding (e.g., ratios of the number of sperm bound to nontreated versus hyaluronidasetreated oocytes). Hyaluronidase (Medicult; $80 \mathrm{U} / \mathrm{ml}$; SPM) was choosen because it did not affect the morphology of the zonae. Some of the cumulus-free fresh and aged IVF and ICSI oocytes were then treated for a 30-sec period with hyaluronidase, after which they were washed four times in IVF medium.

For the ZBT, oocytes were then inseminated in IVF medium with $10^{5}$ motile spermatozoa/ml. After $4 \mathrm{hr}$ of incubation at $37^{\circ} \mathrm{C}$ with $5 \% \mathrm{CO}_{2}$ in air, oocytes were vigorously washed by pipetting (10 times) to release loosely bound spermatozoa. Tightly bound spermatozoa were then counted in an inverted Nikon microscope equipped with a heated stage and Hoffman modulation contrast optics. Only high-quality (with a homogeneous cytoplasmic appearance, without granularities or vacuoles) mature oocytes, with similar zona thicknesses (12-15 $\mu \mathrm{m})$ and surface appearances, were used, and each ZBT (coupling) was performed only with oocytes from the same woman and sperm from the same donor. Because IVF oocytes contained previously bound spermatozoa in their zonae, these were counted first before performing the ZBT. The different number of eggs used for control and treated groups is due to the fact that, when there was an odd number of eggs present, more eggs were ascribed to the treated group.

The data are presented as mean \pm SD. For assessing the influence of oocyte pretreatment within each group, a paired Student's $t$ test was performed. The effect of the different pretreatments among groups was first examined by analysis of variance (ANOVA). After that, specific differences between groups were determined by post hoc comparisons. All the performed tests were two-tailed and the differences were considered non significant at $P>0.05$. Statistical analysis were performed with the computer programs Excel and Statistica (Versions 4.0).

\section{RESULTS}

\section{Experimental Group I}

To investigate if hyaluronidase digestion of the zona pellucida affects sperm binding, 7 individual ZBTs (couplings) were performed in a total of 26 fresh oocytes which were previously freed of their cumulus layers by mechanical means. Ten of these oocytes were left undisturbed (control oocytes), while the other 16 were further digested with hyaluronidase (treated oocytes). Results showed that this treatment significantly decreased the binding of sperm to the zona (Table I).

\section{Experimental Group II}

To investigate if aging affects sperm binding, 10 individual ZBTs (couplings) were performed in a total of 67 fresh oocytes which were left in culture for $44 \mathrm{hr}$ before being freed of their cumulus layers by mechanical means. Twenty-nine of these experimentally aged oocytes were left undisturbed (control oocytes), while the other 38 were further digested with 
Table I. Effect of Oocyte Pretreatment on the Mean Total Number of Spermatozoa Linked to the Zona Pellucida

\begin{tabular}{lccccc}
\hline & Group I & Group II & Group III $^{e}$ & Group IV $^{d}$ & Group V $^{g}$ \\
\hline Number of couplings $^{b}$ & 7 & 10 & 9 & 13 & 4 \\
Sperm per control oocyte & $32.7 \pm 20.6$ & $35.0 \pm 24.6$ & $49.6 \pm 70.8$ & $81.5 \pm 92.5$ & $29.0 \pm 17.0$ \\
Sperm per treated oocyte & $9.0 \pm 7.4$ & $14.5 \pm 11.5$ & $50.6 \pm 70.8$ & $22.8 \pm 22.5$ & $8.3 \pm 4.2$ \\
Mean difference of control vs treated & $23.7 \pm 14.1$ & $21.6 \pm 17.1$ & $-1.0 \pm 2.5$ & $58.8 \pm 70.4$ & $20.8 \pm 13.5$ \\
$\quad$ & $P=0.004$ & $P=0.003$ & $P>0.05$ & $P=0.01$ & $P=0.05$ \\
\hline
\end{tabular}

${ }^{a}$ Values are means \pm SD.

${ }^{b}$ In each ZBT (coupling), only oocytes and sperm from the same patient were used.

c Fresh oocytes freed of their cumulus by mechanical means.

${ }^{d}$ Fresh oocytes left to age for $44 \mathrm{hr}$ and then freed of their cumulus by mechanical means.

e Fresh oocytes freed of their cumulus either mechanically (control) or by hyaluronidase treatments (treated).

$f$ Aged unfertilized ICSI oocytes.

Aged unfertilized IVF oocytes.

${ }^{h}$ Except for Group III, treated oocytes were those whose zonae pellucidae were digested with hyaluronidase.

' $P$ levels for the significance of the difference between the means of couplings within each group, by paired Student's $t$ test.

hyaluronidase (treated oocytes). Results showed that aging did not affect the significant hyaluronidaseinduced decrease in sperm binding (Table I).

\section{Experimental Group III}

To analyze if hyaluronidase removal of the cumulus layer affects sperm binding, we performed 9 individual ZBTs (couplings) in a total of 44 fresh oocytes. In 20 of these cells the cumulus layer was removed mechanically (control oocytes), while in the other 24 oocytes it was removed by hyaluronidase treatment (treated oocytes). Results showed that this treatment did not influence sperm-zona binding (Table I).

\section{Experimental Group IV}

In this group we investigated how hyaluronidase digestion of the zona pellucida affects sperm binding in unfertilized aged ICSI oocytes. Thirteen individual ZBTs (couplings) were performed in a total of 47 unfertilized aged ICSI oocytes. Twenty-one of these cells were left undisturbed (control oocytes), whereas the other 26 were further treated with hyaluronidase (treated oocytes). Results showed that this treatment also significantly reduced sperm-zona binding (Table I).

\section{Experimental Group V}

In this group we investigated how hyaluronidase digestion of the zona pellucida affects sperm binding in unfertilized aged IVF oocytes. Four individual ZBTs (couplings) were performed in a total of 23 unfertilized aged oocytes. Nine of these cells were left undisturbed (control oocytes), whereas the other 14 were further treated with hyaluronidase (treated oocytes). Results showed that this treatment also significantly reduced sperm-zona binding (Table I).

\section{Between Group Analysis}

According to the paired analysis within groups (Table I), the pretreatment (hyaluronidase digestion of the zona pellucida) was more effective in fresh and in experimentally aged oocytes (Groups I and II) than in unfertilized aged oocytes from ICSI (Group IV) or IVF (Group V) cycles, the levels of significance of the differences being lower for Group V than for Group IV. However, the between-group analysis (Table II) showed that this order of effectiveness is an apparent one when we rely on the mean ratio as a success index (e.g., ratio of the number of sperm bound to nontreated versus treated oocytes). This index showed that hyaluronidase digestion of the zona pellucida decreased the ratio of sperm binding to the zona pellucida by three to four times in Groups I, II, IV, and V, with no statistical significant differences being noticed between these groups (Table II).

\section{DISCUSSION}

The present study shows that unstored aged mature unfertilized ICSI oocytes have the same sperm binding potential as fresh oocytes under intact-zona assay conditions. Similar results were obtained with aged IVF 
Table II. Effect of Oocyte Pretreatment on the Mean Ratio Between the Number of Spermatozoa Linked to the Zona Pellucida of a Control versus of a Treated Oocyte ${ }^{a}$

\begin{tabular}{|c|c|c|c|c|}
\hline & Group I & Group II & Group IV & Group V \\
\hline Number of couplings ${ }^{b}$ & 7 & 10 & 13 & 4 \\
\hline Ratio control/treated ${ }^{b}$ & $4.21 \pm 1.85$ & $3.21 \pm 3.08$ & $3.30 \pm 0.66$ & $3.43 \pm 0.85$ \\
\hline
\end{tabular}

${ }^{a}$ Values are means $\pm S D$.

${ }^{b}$ No significant differences were detected among groups.

oocytes under the same conditions, which were used as a further internal control.

To compare the sperm binding ability of differently prepared oocytes and overcome the problem of the large variation in sperm binding scores, we compared the changes observed in the sperm binding ability of each set of oocytes after priming the zonae with an agent that decreases sperm binding. For this, hyaluronidase was preferred because it did not affect the morphology of the zonae. In this way, each set of oocytes coming from the same patient was divided into a control group (zonae not exposed to hyaluronidase) and a treated group (zonae exposed to hyaluronidase). By this method the sperm binding ability of the different sets of oocytes could be compared by using the ratio of the number of sperm bound to nontreated versus treated oocytes.

It is worth noting that hyaluronidase digestion of the cumulus did not affect sperm binding, contrary to what was found when the enzyme was applied to the zona pellucida. This is explained by the fact that a short exposure $(30 \mathrm{sec})$ of the cumulus to the enzyme allows digestion of only the hyaluronic acid that exists between cumulus cells. In practice, after this digestion, corona cells must then be detached from the zona pellucida by mechanical means, further showing that this short enzyme exposure does not reach as far as the zona.

To decrease the number of variables being analyzed simultaneously, we first studied the influence of aging and of the enzymatical removal of the cumulus by using only fresh oocytes. To study the effect of aging, we determined the sperm binding ability in control and hyaluronidase-treated fresh oocytes after having left them to age for $44 \mathrm{hr}$ in culture. To determine if hyaluronidase removal of the cumulus layer could affect the results, we compared the sperm binding scores of fresh mature oocytes in which the cumulus was either mechanically or enzymatically removed. In both studies, results showed that aging (Group II) or enzymatical removal of the cumulus layer (Group III) did not affect the sperm binding ability of the oocytes.
By using these two further controls, we could then compare the sperm binding ability of fresh (Group I) and aged (Group IV) unfertilized ICSI and IVF (Group V) oocytes. When between-group analysis was performed by using the mean ratio of nontreated versus treated oocytes, the sperm-zona binding was found to be decreased by three to four times in all four groups (I, II, IV, and V), which demonstrates that the sperm binding ability of fresh and unfertilized aged ICSI and IVF oocytes is similar.

In conclusion, our results, using unstored mature unfertilized aged oocytes coming from IVF cycles and used under intact-zona assay conditions, confirm the results of others, which have shown that pooling, storage, aging, and previous sperm exposure do not affect sperm binding (1-6, 15-20). Our results expand the present knowledge by showing that hyaluronidase removal of the cumulus layer and microinjection also do not affect sperm binding, thus showing that aged mature unfertilized ICSI oocytes can also be used safely for tests of sperm binding.

\section{ACKNOWLEDGMENTS}

This work was partially supported by JNICT and the Eng. António de Almeida Foundation. We thank Mrs. Elsa Oliveira for technical expertise.

\section{REFERENCES}

1. Franken DR, Coddington CC, Burkman LJ, Oosthuizen WT, Oehninger SC, Kruger TF, Hodgen GD: Defining the valid hemizona assay: Accounting for binding variability within zonae pellucidae and within semen samples from fertile males. Fertil Steril 1991;56:1156-1161

2. Gamzu R, Yogev L, Yavetz H, Homonnai ZT, Hiss Y, Paz G: Fresh and frozen-thawed human sperm bind in a similar pattern to the zona pellucida in the hemizona assay. Fertil Steril 1992;58:1254-1256

3. Oehninger S, Mahony MC, Swanson JR, Hodgen GD: The specificity of human spermatozoa/zona pellucida interaction 
under hemizona assay conditions. Mol Reprod Dev 1993; 35:57-61

4. Liu DY, Lopata A, Johnston WIH, Baker HWG: A human sperm-zona pellucida binding test using oocytes that failed to fertilize in vitro. Fertil Steril 1988;50:782-788

5. Morroll DR, Critchlow JD, Matson PL, Lieberman BA: The use of cryopreserved aged human oocytes in a test of the fertilizing capacity of human spermatozoa. Hum Reprod 1992;7:671-676

6. Graczykowski JW, Francis M, Paulson RJ, Sokol RZ: Variability of sperm-zona pellucida binding in vitro using the intact zona model. Abstr 44th Ann Meet Pacific Coast Fertil Soc, 1996, A19

7. Oehninger S, Acosta A, Hodgen GD: Antagonistic and agonistic properties of saccharide moieties in the hemizona assay. Fertil Steril 1990;53:143-149

8. Oehninger S, Clark GF, Acosta AA, Hodgen GD: Nature of the inhibitory effect of complex saccharide moieties on the tight binding of human spermatozoa to the human zona pellucida. Fertil Steril 1991;55:165-169

9. Oehninger S, Clark GF, Fulgham D, Blackmore PF, Mahony MC, Acosta AA, Hodgen GD: Effect of fucoidin on humanzona pellucida interactions. J Androl 1992;13:519-525

10. Mahony MC, Oehninger S, Clark GF, Acosta AA, Hodgen GD: Fucoidin inhibits the zona pellucida-induced acrosome reaction in human spermatozoa. Contraception 1991;44: 657-665

11. Mahony MC, Blackmore PF, Bronson RA, Alexander NJ: Inhibition of human sperm-zona pellucida tight binding in the presence of antisperm antibody positive polyclonal patient sera. J Reprod Immunol 1991;19:287-301

12. Mahony MC, Fulgham DL, Blackmore PF, Alexander NJ: Evaluation of human sperm-zona pellucida tight binding by presence of monoclonal antibodies to sperm antigens. J Reprod Immunol 1991;19:269-285

13. Tesarik J, Mendoza C, Ramirez J-P, Moos J: Solubilized human zona pellucida competes with a fucosylated neoglycoprotein for binding sites on the human sperm surface. Fertil Steril $1993 ; 60: 344-350$

14. Lassalle B, Testart J: Human zona pellucida recognition associated with removal of sialic acid from human sperm surface. J Reprod Fertil 1994;101:703-711
15. Franken DR, Burkman LJ, Oehninger SC, Coddington CC, Veeck LL, Kruger TF, Rosenwaks Z, Hodgen GD: Hemizona assay using sait-stored human oocytes: evaluation of zona pellucida capacity for binding human spermatozoa. Gamete Res 1989;22:15-26

16. Franken DR, Windr ML, Kruger TF, Oehninger S, Hodgen $\mathrm{GD}$ : Comparison of sperm binding potential of uninseminated, inseminated-unfertilized, and fertilized-noncleaved human oocytes under hemizona assay conditions. Mol Reprod Dev $1991 ; 30: 56-61$

17. Franken DR, Acosta AA, Kruger TF, Lombard CJ, Oehninger $S$, Hodgen GD: The hemizona assay: its role in identifying male factor infertility in assisted reproduction. Fertil Steril 1993;59: 1075-1080

18. Franken DR, Kruger TF, Oehninger S, Coddington CC, Lombard C, Smith K, Hodgen GD: The ability of the hemizona assay to predict human fertilization in different and consecutive in-vitro fertilization cycles. Hum Reprod 1993;8:1240-1244

19. Liu DY, Lopata A, Johnston WIH, Baker HWG: Human spermzona pellucida binding, sperm characteristics and in vitro fertilization. Hum Reprod 1989;4:696-701

20. Oehninger S, Coddington CC, Scott R, Franken DA, Burkman LJ, Acosta AA, Hodgen GD: Hemizona assay: Assessment of sperm dysfunction and prediction of in vitro fertilization outcome. Fertil Steril 1989;51:665-670

21. Van Steirteghem AC, Liu J, Joris H, Nagy Z, Janssenswillen C, Toumaye H, Derde MP, Van Assche E, Devroey P: Higher success rate by intracytoplasmic sperm injection than by subzonal insertion. Report of a second series of 300 consecutive treatment cycles. Hum Reprod 1993;8:1055-1060

22. Van Steirteghem AC, Nagy Z, Joris H, Liu J, Staessen C, Smitz $\mathrm{J}$, Wisanto A, Devroey P: High fertilization and implantation rates after intracytoplasmic sperm injection. Hum Reprod 1993;8:1061-1066

23. Tesarik J, Sousa M, Testart J: Human oocyte activation after intracytoplasmic sperm injection. Hum Reprod 1994;9: $511-518$

24. Tesarik J, Sousa M: Key elements of a highly efficient intracytoplasmic sperm injection technique: $\mathrm{Ca}^{2+}$ fluxes and oocyte cytoplasmic dislocation. Fertil Steril 1995;64:770-776

25. World Health Organization: WHO Laboratory Manual for the Examination of Human Semen and Sperm-Cervical Mucus Interaction. Cambridge, Cambridge University Press, 1992 